\title{
Atrio-ventricular block after administration of lignocaine in patients treated with prenylamine
}

\author{
E. GRENADIER \\ M.D. \\ S. KEIDAR \\ M.D.
}

G. Alpan

M.D.

A. Palant

M.D.

Kupat Holim, Lady Davis Carmel Hospital, Department of Cardiology, Haifa, Israel

\begin{abstract}
Summary
Four patients are described who exhibited prolonged Q-T intervals, multiple ventricular premature beats and ventricular tachycardia of the 'torsade de pointes' configuration whilst on treatment with prenylamine. In two of these patients, advanced atrio-ventricular block was established immediately following intravenous administration of lignocaine. The arrhythmia and the atrio-ventricular blocks were successfully controlled by ventricular pacing. The hazard of the simultaneous administration of these two drugs is stressed.
\end{abstract}

\section{Introduction}

A number of patients with angina treated with prenylamine have recently been seen who exhibited prolonged Q-T intervals, multiple ventricular premature beats, and ventricular tachycardia of the 'torsade de pointes' configuration with syncope. In four such patients, treatment with lignocaine was initiated and was followed by advanced atrioventricular (AV) block in two of them. The arrhythmias were eventually controlled by rapid ventricular pacing but the occurrence of AV block in two out of four patients treated with lignocaine seemed most unusual, and it is to this phenomenon that the authors wish to draw attention.

\section{Case reports}

Patient 1

An 82-year-old man, with stable angina following a previous myocardial infarction had been treated with sublingual nitrates and prenylamine $240 \mathrm{mg} /$ day for several weeks before he was admitted for investigation of complaints of several episodes of syncope. His blood pressure was $160 / 90 \mathrm{mmHg}$ and the pulse was irregular at 59 beats/min. Physical examination revealed no abnormality, and serum electrolytes, calcium, magnesium and liver and kidney function tests were all normal. His electrocardiogram (ECG) showed sinus rhythm with frequent premature ventricular beats, a prolonged Q-T interval $\left(\mathrm{QT}_{\mathrm{c}}-0.64 \mathrm{~s}\right)$ and short runs of ventricular tachycardia exhibiting the 'torsade de pointes' configuration (Fig. 1). An intravenous (i.v.) bolus of $100 \mathrm{mg}$ lignocaine was given and was followed by complete AV block (Fig. 2). A ventricular pacemaker was inserted followed by the patient's immediate recovery. Five days after cessation of prenylamine, the Q-T interval returned to normal, together with complete disappearance of all ventricular ectopics.

\section{Patient 2}

A 76-year-old woman had been treated with sublingual nitrates and prenylamine $240 \mathrm{mg}$ /day for a stable angina. Following several episodes of syncope over the previous 8 weeks she was admitted for continuous monitoring. Physical examination and laboratory investigations revealed no abnormalities. Her ECG showed sinus bradycardia of 40 beats/ min and a prolonged Q-T interval $\left(\mathrm{QT}_{\mathrm{c}}-0.61 \mathrm{~s}\right)$. Ventricular premature beats were observed accompanied by the torsade de pointes ventricular tachycardia. Following administration of a $50 \mathrm{mg}$ i.v. bolus of lignocaine, the patient developed Mobitz II $^{\circ}$ AV block (Fig. 3). A ventricular pacemaker was inserted and subsequent runs of arrhythmias were controlled by overdrive suppression. The AV block abated spontaneously after several minutes. Three days after cessation of prenylamine the Q-T interval returned to normal without recurrence of ventricular ectopic arrhythmias.

In 2 other patients with prenylamine induced ventricular tachycardia, lignocaine did not produce AV conduction delays but did not terminate the 


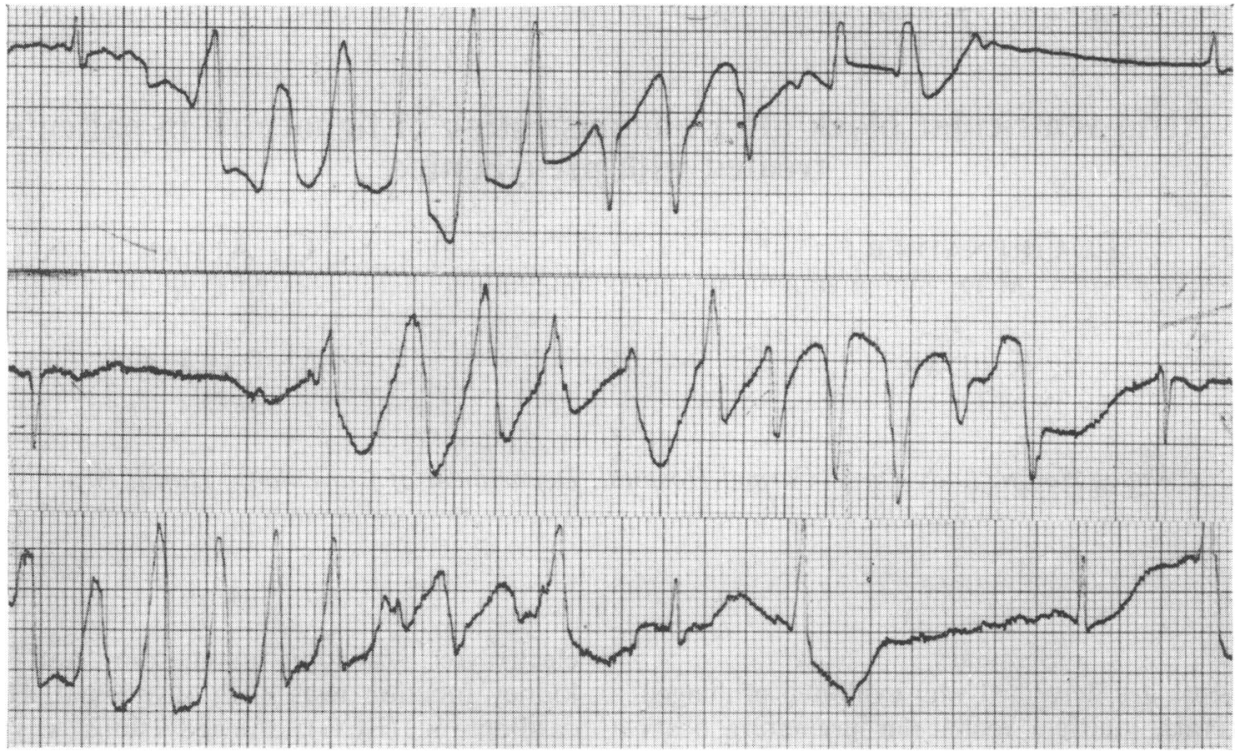

FIG. 1. A run of ventricular tachycardia exhibiting the torsade de pointes phenomenon, patient 1.

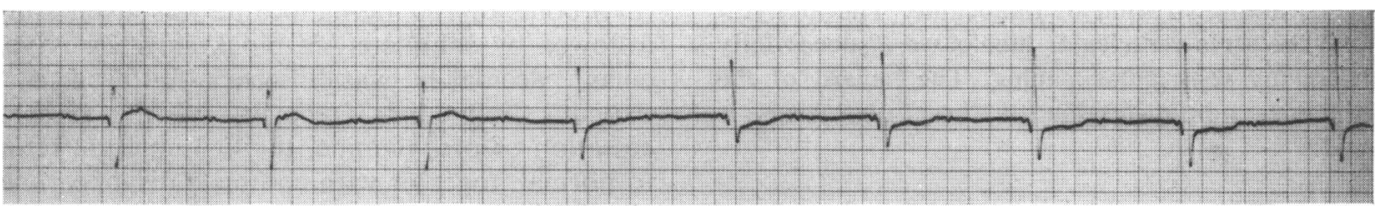

FIG. 2. Complete AV block (after i.v. administration of a $100 \mathrm{mg}$ bolus of lignocaine).

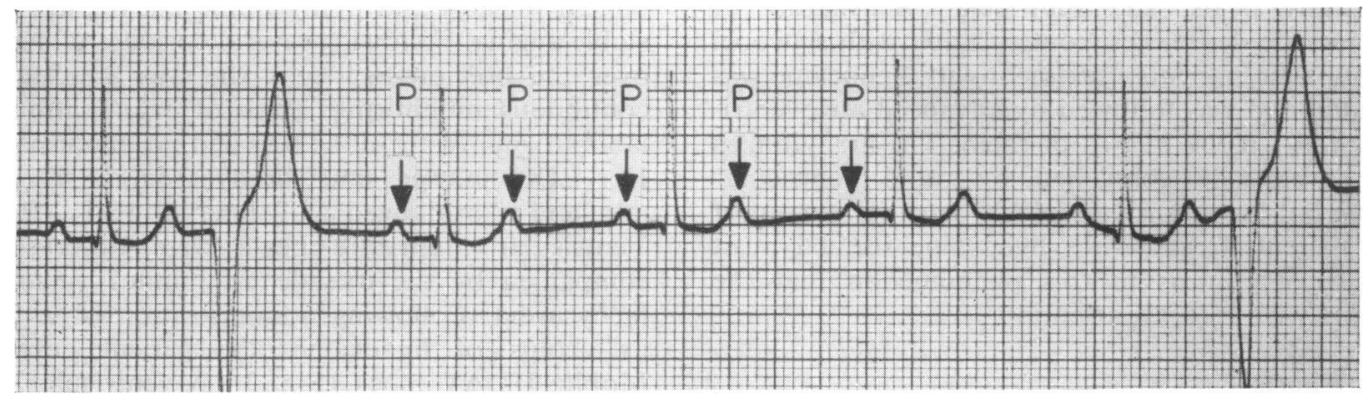

FIG- 3. 1:2 AV block (after intravenous administration of a $50 \mathrm{mg}$ bolus of lignocaine).

arrhythmias, which were treated by pacemaker overdrive suppression. On the basis of this experience it has been the authors'policy in subsequent cases to insert a pacemaker electrode as treatment of first choice.

\section{Discussion}

AV block after intravenous administration of lignocaine is an extremely rare occurrence. In over 1000 patients treated with lignocaine at the Lady Davis Carmel Hospital, the cases mentioned above 
are the only ones to have exhibited conduction delays following a bolus injection. In the normal cardiac conduction system, administration of lignocaine has no effect or at most causes a very slight delay on the P-A or A-H intervals, duration of the QRS or the Q-T intervals (Ryden, Culhed and Wasir, 1972; Rosen, Lam and Weiss, 1970). Patients with advanced AV block or old existing blocks also rarely develop complete heart block following treatment with lignocaine (Kunkel, Rowland and Scheinman, 1974; Bekheit, Murtagh and Morton, 1973; Gupta, Lichstein and Chadd, 1974). The few cases described in the literature of AV blocks following an i.v. bolus of lignocaine have all occurred in patients with previous gross conduction defects (Gianelly, Van der Groben and Spiral, 1967; Josephson, Caracta and Lam, 1972; Liss, Jeresaty and Nakhaul, 1973; Lichstein, (Chadd and Gupta, 1973). Thus, Lichstein et al. (1973) described a patient with a tri-fascicular block who twice developed complete AV block after i.v. administration of $50 \mathrm{mg}$ lignocaine.

Josephson et al. (1972) described a patient with complete left bundle branch block and an $\mathrm{A}-\mathrm{H}$ interval of $50 \mathrm{~ms}$, who developed complete AV block several seconds after an $100 \mathrm{mg}$ bolus of lignocaine was administered. The block was located in the atrioventricular junction.

None of the present four patients had any electrocardiographic evidence of conduction delays or abnormalities before administration of lignocaine. Thus, the occurrence of AV block in 2 of 4 patients treated with lignocaine seems to be attributable to factors other than chance. It is possible that the interaction between prenylamine and lignocaine predisposes to inducement of AV conduction delays by the latter. Prenylamine may cause bradycardia and may also cause a delay of the electrical stimulus in the upper conducting system (Fleccenstein, 1968), although it is not known to cause AV block. Prenylamine may produce prolonged $\mathrm{Q}-\mathrm{T}$ intervals with the induction of polymorphous ventricular tachycardia, accompanied by episodes of syncope (Puvitz et al., 1977). Since the inclination would be to administer lignocaine to control the arrhythmia, the authors wish to draw attention to the possible harm that may evolve of such an action. Although lignocaine is not an effective drug in cases of 'torsade de pointes' ventricular tachycardia in any case, the configuration of this arrhythmia is not always obvious on a monitor lead and it may be mistaken for simple ventricular tachycardia until a multiple lead ECG is taken. Moreover, although ineffective, lignocaine is not known to cause AV block in 'torsade de pointes' ventricular tachycardia. Although their hypothesis rests on circumstantial evidence, the authors think the warning should be kept in mind. Confirmation will depend on further reports of ventricular arrhythmias treated with lignocaine in patients on prenylamine.

\section{References}

Bekheit, S., Murtagh, J.G. \& Morton, P. (1973) Effect of lignocaine on conducting system of human heart. British Heart Journal, 35, 305.

Fleccenstein, A. (1968) Influence of prenylamine on the utilization of high energy phosphates in cardiac muscle. Biochemica applicata, 14 (suppl. 1), 323.

Gianelly, R., VAN DeR Groeben, J.O. \& Spival, A.P. (1967) Effect of lignocaine on ventricular arrhythmias in patients with coronary heart diseases. New England Journal of Medicine, 277, 1215.

Gupta, P.K., Lichstein, E. \& Chadd, K.D. Lignocaineinduced heart block in patients with bundle branch block. American Journal of Cardiology, 33, 487.

Josephson, M.E., Caracta, A.R. \& LAM, S.H. (1972) Effects of lignocaine on refractory periods of man. American Heart Journal, 84, 778.

Kunkel, F., Rowland, M., Scheinman, M.M. (1974) The electrophysiologic effect of lignocaine in patients with intraventricular conduction defects. Circulation, 49, 894.

Lichstein, E., Chadd, K.D. \& Gupta, P.K. (1973) Atrioventricular block with lignocaine therapy. American Journal of Cardiology, 31, 277.

Liss Jr J.P., Jeresaty, R.M. \& Nakhaul, J. (1973) Lignocaine in ventricular arrhythmia. American Heart Journal, 86, 143.

Puvitz, R., Henderson, M.A. Baker, S.N. \& ChamberLAIN, D.A. (1977) Ventricular arrhythmia caused by prenylamine. British Medical Journal, 2, 608.

Rosen, K.M., LAM, S.H. \& WeisS, M.B. (1970) The effect of lignocaine on atrioventricular and intraventricular conduction in man. American Journal of Cardiology, 25, 1-5.

Ryden, L., Culhed, I. \& WasiR, H. (1972) Effect of lignocaine on heart rate in patients with sinus bradycardia associated with proven or suspected acute myocardial infarction. American Journal of Cardiovascular Research, 6, 664 . 